\title{
Optimization of the Upper Surface of Hypersonic Vehicle Based on CFD Analysis
}

\author{
T. Y. Gao, K. Cui*, S. C. Hu, X. P. Wang, G. W. Yang
}

Key Laboratory of High Temperature Gas Dynamics, Institute of Mechanics, Chinese Academy of Science, Beijing 100190, China

Email: Kcui@imech.ac.cn

\begin{abstract}
For the hypersonic vehicle, the aerodynamic performance becomes more intensive. Therefore, it is a significant event to optimize the shape of the hypersonic vehicle to achieve the project demands. It is a key technology to promote the performance of the hypersonic vehicle with the method of shape optimization. Based on the existing vehicle, the optimization to the upper surface of the Simplified hypersonic vehicle was done to obtain a shape which suits the project demand. At the cruising condition, the upper surface was parameterized with the $B$-Spline curve method. The incremental parametric method and the reconstruction technology of the local mesh were applied here. The whole flow field was been calculated and the aerodynamic performance of the craft were obtained by the computational fluid dynamic (CFD) technology. Then the vehicle shape was optimized to achieve the maximum lift-drag ratio at attack angle $3^{\circ}, 4^{\circ}$ and $5^{\circ}$. The results will provide the reference for the practical design.
\end{abstract}

Key words: hypersonic, shape optimization, sequential quadratic programming (SQP)

\section{INTRODUCTION}

As one of the strategic high ground in new era, with huge commercial interests, the development of hypersonic vehicles has important military significance. However, the aerodynamic performance requirement of the vehicles becomes more and more because of the complex phenomena of hypersonic flow. The fore body of hypersonic vehicle not only has the ability to provide the most needed lift but also plays the role as the pre-compression face of the inlet of the engine. The aft body of the vehicle can be considered as the extension of the nozzle, which can provide the lift and thrust. With this design, the lower surface of the vehicle and the propulsion system are integrated into one part. Therefore, it is a significant event to optimize the shape of the hypersonic vehicle to achieve the project demands. It is a key technology to promote the development of the hypersonic vehicle with the method of shape optimization. The shape of the lower surface of the vehicle is decided by the propulsion system. So, it's necessary that designing the upper surface to achieve the maximum lift-drag ratio.

Configuration optimization design of hypersonic vehicle is a new technology, which is a combination of computational fluid dynamics (CFD) technology and numerical optimization method to obtain the optimal design. Sequential quadratic programming (SQP) method was applied to the optimization of the upper surface of the Simplified hypersonic vehicle in order to obtain a configuration which suits the project demand. At the cruising condition, the upper surface was parameterized with the $B$-Spline curve method. The incremental parametric method, CFD analysis and the reconstruction technology of the local mesh were also applied here as tools. In the process of optimization the volume of airframe was used as a constraint condition. The results verify the effectiveness of the incremental parametric method and the optimization process, and provide a basis for further research.

\section{VEHICLE CONFIGURATION}

The shape of the lower surface of the vehicle is decided by the propulsion system. So, it's necessary that designing the upper surface to achieve the maximum lift-drag ratio. Therefore, the simplified configuration does not install the rudder and the propulsion system. The fore body of the vehicle uses wave rider configuration. The length of the vehicle is $6.5 \mathrm{~m}$ long, and the reference area is $2.37 \mathrm{~m}^{2}$, as shown below. 


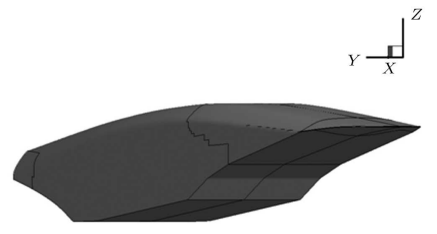

Figure 1: A 3D model of simplified hypersonic vehicle

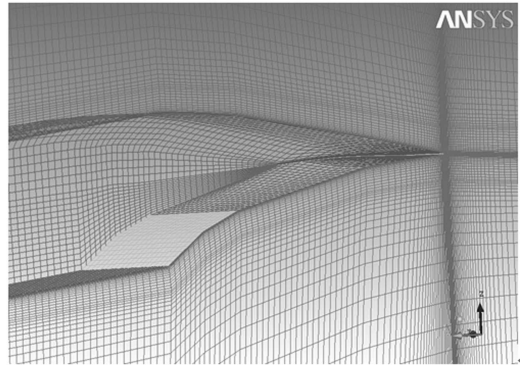

Figure 2: Multi-Zone mesh

\section{Incremental parametric method}

The incremental parametric method is applied to the parameterization of the upper surface. Based on the original surface $A$, the incremental parametric layer $A^{\prime}$ is overlaid on surface $A$ to produce the new parametric surface $\tilde{A}$. In the overlay process, the interpolation technique is applied point to point. Maintain the points on the original and incremental layer have the same $x$-, $y$-coordinate, and overlay the $z$-coordinate.

The BSP curve technique is applied to generate a parametric curve as the symmetry curve of the incremental layer (the $y$-coordinate equal to 0 , the $x z$-section). The $z$-coordinate of the point on this curve is the max incremental coordinate in its $y$-direction of the layer. The incremental coordinate of each point along the $y$-direction change to 0 on the boundary according quadratic curve. Maintain the boundary of the incremental layer coincides with the boundary of original surface. The same process can be done in the $y z$-section to generate the incremental layer along the $x$-direction. Piecewise cubic $B$-spline curve method is applied here, the equation is

$P(t)=\frac{1}{6}\left[\begin{array}{llll}t^{3} & t^{2} & t & 1\end{array}\right]\left[\begin{array}{cccc}-1 & 3 & -3 & 1 \\ 3 & -6 & 3 & 0 \\ -3 & 0 & 3 & 0 \\ 1 & 4 & 1 & 0\end{array}\right]\left[\begin{array}{l}P_{0} \\ P_{1} \\ P_{2} \\ P_{3}\end{array}\right], t \in[0,1]$

And 7 design variables are used to generate the new parametric surface.

\section{COMPUTATIONAL CONDITION}

During the CFD analysis, considering the environmental and the physical characteristics of hypersonic flow, the physical model and boundary conditions we used are as follows,

Physical model: 3 dimensional Navier-Stokes equations, $k-\omega$ turbulence model.

Boundary condition: $M a=6.5, T_{\infty}=223.6 \mathrm{~K}, P_{\infty}=1847.5 \mathrm{pa}, T_{0}=2108.7 \mathrm{~K}$, Attack angle: $3^{\circ}, 4^{\circ}$ and $5^{\circ}$.

All of the results are based on half of the shape.

\section{OPTIMIZATION PROCESS}

In the optimization process, the CFD analysis is done first to gain the aerodynamic parameters of every shape. Then the design variables are changed under the guidance of optimization method to generate the new shape. After the local mesh reconstruction the further CFD analysis will be done automatically. The process will not stop until the aerodynamic parameters meet requirements.

The SQP method is used in the current optimization process. The main idea of this method is transforming the optimization problem into a series of quadratic programming sub-problems solved by iteration. SQP method is one of the most robust methods for solving the nonlinear optimization problems.

The optimization target is the maximum lift-drag ratio of the vehicle. In the process of optimization the volume of airframe is used as a constraint condition. The specific results are shown in this paper.

\section{RESULTS}

The comparison between the initial and the optimized design variables are listed in Table 1. $X$-Init represents the initial variables, while $X$-Optm represents the optimized variables. 
Table 1. Comparison of initial and optimized design variables

\begin{tabular}{cccccc}
\hline \multicolumn{2}{c}{7 variables $\left(3^{\circ}\right)$} & \multicolumn{2}{c}{7 variables $\left(4^{\circ}\right)$} & \multicolumn{2}{c}{7 variables $\left(5^{\circ}\right)$} \\
\hline$X$-Init & $X$-Optm & $X$-Init & $X$-Optm & $X$-Init & $X$-Optm \\
\hline 0.06 & $-6.6594033 \times 10^{-2}$ & 0.06 & $-4.6238046 \times 10^{-2}$ & 0.06 & $-1.8003795 \times 10^{-1}$ \\
0.06 & $-8.6187647 \times 10^{-2}$ & 0.06 & $-8.7626528 \times 10^{-2}$ & 0.06 & $-3.4766348 \times 10^{-2}$ \\
0.06 & $-6.1380820 \times 10^{-2}$ & 0.06 & $-4.7536013 \times 10^{-2}$ & 0.06 & $1.0000000 \times 10^{-1}$ \\
0.06 & $-7.7865630 \times 10^{-2}$ & 0.06 & $-3.6555136 \times 10^{-2}$ & 0.06 & $-1.0000000 \times 10^{-1}$ \\
0.06 & $-2.5276257 \times 10^{-2}$ & 0.06 & $-8.9396544 \times 10^{-2}$ & 0.06 & $-1.0000000 \times 10^{-1}$ \\
0.06 & $-9.0933955 \times 10^{-2}$ & 0.06 & $-1.0000000 \times 10^{-1}$ & 0.06 & $-9.7289485 \times 10^{-2}$ \\
0.005 & $1.8292181 \times 10^{-2}$ & 0.005 & $1.9061206 \times 10^{-2}$ & 0.005 & $4.5000000 \times 10^{-2}$ \\
\hline
\end{tabular}

The comparison between the initial and the optimized aerodynamic parameters are listed in Table 2 . $X$-Init represents the initial parameters, while $X$-Optm represents the optimized parameters. $C_{\mathrm{d}}$ represents the drag coefficient, $C_{1}$ represents the lift coefficient and the $C_{\mathrm{l}} / C_{\mathrm{d}}$ represents the lift-drag ratio. $\Delta C_{\mathrm{d}}$ represents the increment of drag coefficient after optimization, $\Delta C_{1}$ represents the increment of lift coefficient after optimization, and $\Delta C_{1} / C_{\mathrm{d}}$ represents the increment of lift-drag ratio after optimization.

The results in the Table 2 indicate the optimization process works well. At $3^{\circ}$ attack angle, the increment of the lift-drag ratio reaches $11.57 \%$. With the increases of the attack angle, the increment of lift-drag ratio falls down to 5.66\%. This is because leeward side effects more obvious at higher attack angle. The volumes of all the three optimized airframe meet the limit $1.7 \mathrm{~m}^{3}$.

Table 2. Comparison of aerodynamic parameters before and after optimization

\begin{tabular}{|c|c|c|c|c|c|c|c|c|c|c|c|}
\hline \multirow{2}{*}{ At lack angle of 7 variables } & \multicolumn{2}{|c|}{$\overline{C_{\mathrm{d}}}$} & \multirow{2}{*}{$\frac{\Delta C_{\mathrm{d}}}{\text { Init Optm }}$} & \multicolumn{2}{|c|}{$\overline{C_{1}}$} & \multirow{2}{*}{$\frac{\Delta C_{1}}{\text { Init Optm }}$} & \multicolumn{2}{|c|}{$\overline{C_{\mathrm{l}} / C_{\mathrm{d}}}$} & \multirow{2}{*}{$\frac{\Delta C_{\mathrm{l}} / C_{\mathrm{d}}}{\text { Init Optm }}$} & \multicolumn{2}{|c|}{ Volume } \\
\hline & Init & Optm & & Init & Optm & & Init & Optm & & Init & $\overline{O p t m}$ \\
\hline $3^{\circ}$ & 0.0272 & 0.0258 & $-4.91 \%$ & 0.0808 & 0.0858 & $6.09 \%$ & 2.9750 & 3.3192 & $11.57 \%$ & 2.1639 & 1.7003 \\
\hline $4^{\circ}$ & 0.0313 & 0.0303 & $-3.31 \%$ & 0.0939 & 0.0989 & $5.32 \%$ & 2.9960 & 3.2635 & $8.93 \%$ & 2.1639 & 1.7000 \\
\hline $5^{\circ}$ & 0.0361 & 0.0355 & $-1.74 \%$ & 0.1073 & 0.1114 & $3.82 \%$ & 2.9726 & 3.1409 & $5.66 \%$ & 2.1639 & 1.7597 \\
\hline
\end{tabular}

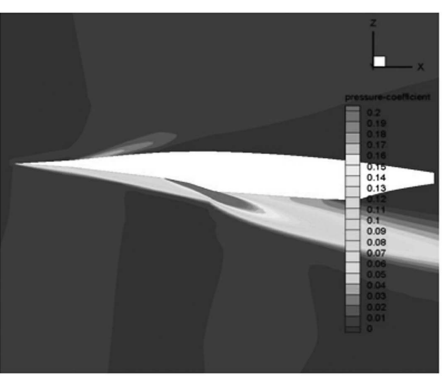

(a) Before optimization

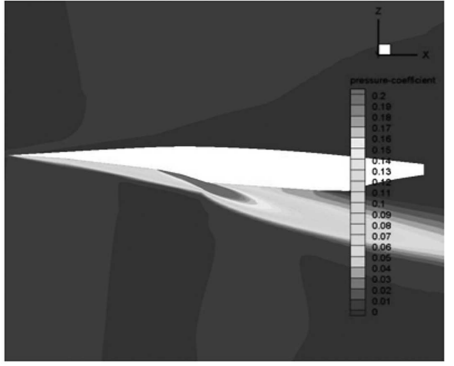

(b) After optimization

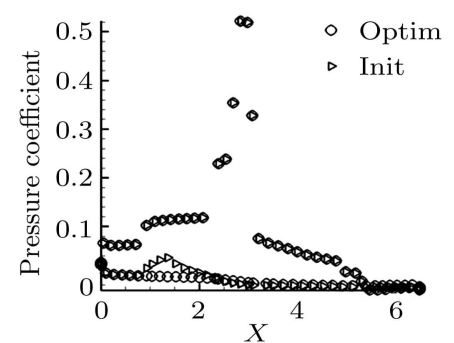

(c) Pressure coefficient of the wall along the symmetry plane

Figure 3: Comparison of the pressure contours on the symmetry plane before and after optimization

The comparison of the pressure contours on the symmetry plane between the initial shape and the optimized shape are given in Figure 3. The figure on the right gives the pressure coefficient of the wall along the symmetry plane. It can be found that the pressure distribution on the upper surface is more uniform after optimization. This indicates that the optimization works well.

\section{Acknowledgements}

The support of National Natural Science Foundation of China (90916013) is gratefully acknowledged.

\section{REFERENCES}

1. Cui K, Yang G W. Shape optimization for hypersonic arc-wing missiles. Journal of Spacecraft and Rockets., 2010; 47(4): 694-700

2. Kevin G.Bowcutt, Geojoe Kuruvila, et al. Advancements in Multidisciplinary Design Optimization Applied to Hypersonic Vehicles to Achieve Closure. 15th AIAA International Space Planes and Hypersonic Systems and Technologies Conference, 28 April-1 May 2008, Dayton, Ohio, AIAA 2008-2591

3. Jing $\mathrm{C}$, Shuo T. Research on integrated optimization design of hypersonic cruise vehile. Aerospace Science and Technology, 2008; 12: 567-572

4. Atsushi Ueno, Kojiro Suzuki. CFD-based Shape Optimization of Hypersonic Vehicles Considering Transonic Aerodynamic Performance. 46th AIAA Aerospace Sciences Meeting and Exhibit, 7-10 January 2008, Reno, Nevada, AIAA 2008-288 